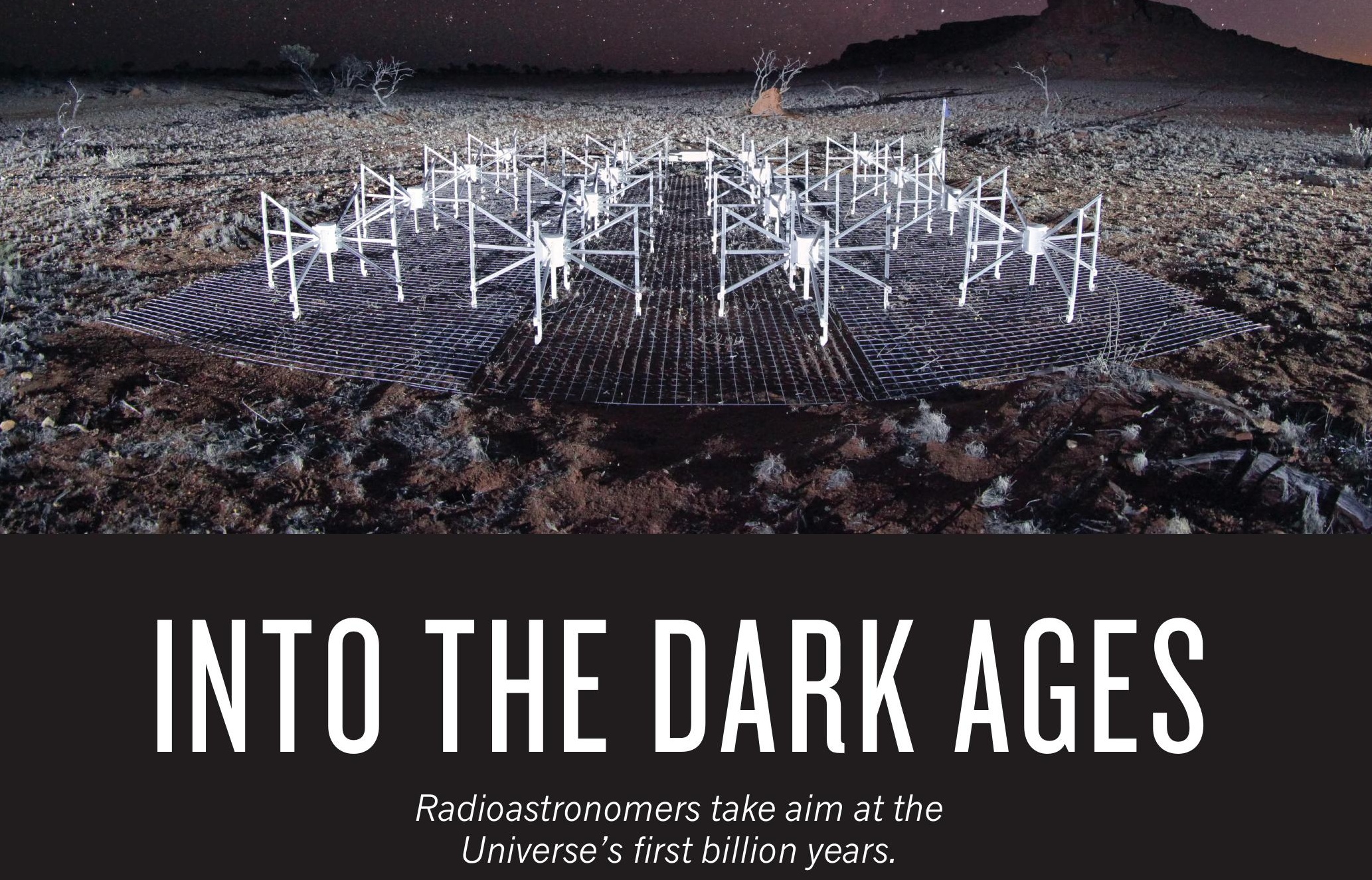

BY DAVIDE CASTELVECCHI

T o get an idea of what the Universe looks like from Earth's perspective, picture a big watermelon. Our Galaxy, the Milky Way, is one of the seeds, at the centre of the fruit. The space around it, the pink flesh, is sprinkled with countless other seeds. Those are also galaxies that we - living inside that central seed — can observe through our telescopes.

Because light travels at a finite speed, we see other galaxies as they were in the past. The seeds farthest from the centre of the watermelon are the earliest galaxies seen so far, dating back to a time when the Universe was just one-thirtieth of its current age of 13.8 billion years. Beyond those, at the thin, green outer layer of the watermelon skin, lies something primeval from before the time of stars. This layer represents the Universe when it was a mere 380,000 years old, and still a warm, glowing soup of subatomic particles. We know about that period because its light still ripples through space - although it has stretched so much over the eons that it now exists as a faint glow of microwave radiation.

The most mysterious part of the observable 
A night view of part of the Murchison Widefield Array in Western Australia.
Universe is another layer of the watermelon, the section between the green shell and the pink flesh. This represents the first billion years of the Universe's history (see 'An Earth's-eye view of the early Universe'). Astronomers have seen very little of this period, except for a few, exceedingly bright galaxies and other objects.

Yet this was the time when the Universe underwent its most dramatic changes. We know the end product of that transition - we are here, after all — but not how it happened. How and when did the first stars form, and what did they look like? What part did black holes play in shaping galaxies? And what is the nature of dark matter, which vastly outweighs ordinary matter and is thought to have shaped much of the Universe's evolution?

An army of radioastronomy projects small and large is now trying to chart this terra incognita. Astronomers have one simple source of information - a single, isolated wavelength emitted and absorbed by atomic hydrogen, the element that made up almost all ordinary matter after the Big Bang. The effort to detect this subtle signal - a line in the spectrum of hydrogen with a wavelength of 21 centimetres - is driving astronomers to deploy ever-moresensitive observatories in some of the world's most remote places, including an isolated raft on a lake on the Tibetan Plateau and an island in the Canadian Arctic.

Last year, the Experiment to Detect the Global Epoch of Reionization Signature (EDGES), a disarmingly simple antenna in the Australian outback, might have seen the first hint of the presence of primordial hydrogen around the earliest stars ${ }^{1}$. Other experiments are now on the brink of reaching the sensitivity that's required to start mapping the primordial hydrogen - and therefore the early Universe in 3D. This is now the "last frontier of cosmology", says theoretical astrophysicist Avi Loeb at the Harvard-Smithsonian Center for Astrophysics (CfA) in Cambridge, Massachusetts. It holds the key to revealing how an undistinguished, uniform mass of particles evolved into stars, galaxies and planets. "This is part of our genesis story - our roots," says Loeb.

\section{A FINE LINE}

Some 380,000 years after the Big Bang, the Universe had expanded and cooled enough for its broth of mostly protons and electrons to combine into atoms. Hydrogen dominated ordinary matter at the time, but it neither emits nor absorbs photons across the vast majority of the electromagnetic spectrum. As a result, it is largely invisible.

But hydrogen's single electron offers an exception. When the electron switches between two orientations, it releases or absorbs a photon. The two states have almost identical energies, so the difference that the photon makes up is quite small. As a result, the photon has a relatively low electromagnetic frequency and so a rather long wavelength, of slightly more than $21 \mathrm{~cm}$.

It was this hydrogen signature that, in the 1950s, revealed the Milky Way's spiral structure. By the late 1960s, Soviet cosmologist Rashid Sunyaev, now at the Max Planck Institute for Astrophysics in Garching, Germany, was among the first researchers to realize that the line could also be used to study the primordial cosmos. Stretched, or redshifted, by the Universe's expansion, those 21-cm photons would today have wavelengths ranging roughly between 1.5 and 20 metres corresponding to 15-200 megahertz (MHz).

Sunyaev and his mentor, the late Yakov Zeldovich, thought of using the primordial hydrogen signal to test some early theories for how galaxies formed ${ }^{2}$. But, he tells Nature, "When I went to radioastronomers with this, they said, 'Rashid, you are crazy! We will never be able to observe this."

The problem was that the hydrogen line, redshifted deeper into the radio spectrum, would be so weak that it seemed impossible to isolate from the cacophony of radio-frequency signals emanating from the Milky Way and from human activity, including FM radio stations and cars' spark plugs.

The idea of mapping the early Universe with $21-\mathrm{cm}$ photons received only sporadic attention for three decades, but technological advancements in the past few years have made the technique look more tractable. The basics of radio detection remain the same;

\section{"THIS IS \\ PART OF OUR GENESIS STORY."}

many radio telescopes are constructed from simple materials, such as plastic pipes and wire mesh. But the signal-processing capabilities of the telescopes have become much more advanced. Consumer-electronics components that were originally developed for gaming and mobile phones now allow observatories to crunch enormous amounts of data with relatively little investment. Meanwhile, theoretical cosmologists have been making a more detailed and compelling case for the promise of 21-cm cosmology.

\section{DARKNESS AND DAWN}

Right after atomic hydrogen formed in the aftermath of the Big Bang, the only light in the cosmos was that which reaches Earth today as faint, long-wavelength radiation coming from all directions - a signal known as the cosmic microwave background (CMB). Some 14 billion years ago, this afterglow of the Big Bang would have looked uniformly orange to human eyes. Then the sky would have reddened, before slowly dimming into pitch darkness; there was simply nothing else there to produce visible light, as the wavelengths of the background radiation continued to stretch through the infrared spectrum and beyond. Cosmologists call this period the dark ages.

Over time, theorists reckon that the evolving Universe would have left three distinct imprints on the hydrogen that filled space. The first event would have begun some 5 million years after the Big Bang, when the hydrogen became cool enough to absorb more of the background radiation than it emitted. Evidence of this period should be detectable today in the CMB spectrum as a dip in intensity at a certain wavelength, a feature that has been dubbed the dark-ages trough.

A second change arose some 200 million years later, after matter had clumped together enough to create the first stars and galaxies. This 'cosmic dawn' released ultraviolet radiation into intergalactic space, which made the hydrogen there more receptive to absorbing $21-\mathrm{cm}$ photons. As a result, astronomers expect to see a second dip, or trough, in the CMB spectrum at a different, shorter wavelength; this is the signature that EDGES seems to have detected ${ }^{1}$.

Half a billion years into the Universe's existence, hydrogen would have gone through an even more dramatic change. The ultraviolet radiation from stars and galaxies would have brightened enough to cause the Universe's hydrogen to fluoresce, turning it into a glowing source of $21-\mathrm{cm}$ photons. But the hydrogen closest to those early galaxies absorbed so much energy that it lost its electrons and went dark. Those dark, ionized bubbles grew bigger over roughly half a billion years, as galaxies grew and merged, leaving less and less luminous hydrogen between them. Even today, the vast majority of the Universe's hydrogen remains ionized. Cosmologists call this transition the epoch of reionization, or EOR.

The EOR is the period that many $21-\mathrm{cm}$ radioastronomy experiments, either ongoing or in preparation, are aiming to detect. The hope is to map it in 3D as it evolved over time, by taking snapshots of the sky at different wavelengths, or redshifts. "We'll be able to build up a whole movie," says Emma Chapman, an astrophysicist at Imperial College London. Details of when the bubbles formed, their shapes and how fast they grew will reveal how galaxies formed and what kind of light they produced. If stars did most of the reionization, the bubbles will have neat, regular shapes, Chapman says. But "if there are a lot of black holes, they start to get larger and more free-form, or wispy", she says, because radiation in the jets that shoot out from black holes is more energetic and penetrating than that from stars.

The EOR will also provide an unprecedented test for the current best model of cosmic evolution. Although there is plenty of evidence for 


\section{AN EARTH'S-EYE VIEW OF THE EARLY UNIVERSE}

The deeper astronomers look into the night sky, the further back in time they see. The oldest observable light is the cosmic microwave background $(\mathrm{CMB})$ - radiation left over from the Big Bang that was emitted when the Universe was just 380,000 years old. Atomic hydrogen formed at that time, and researchers can follow its activities in the early Universe by looking for signs of the radiation that it emitted or absorbed. Hydrogen does this at a characteristic 21-centimetre wavelength, and that radiation has stretched over time as the Universe has expanded. Evidence of that $21-\mathrm{cm}$ signal charts the evolution of the Universe from the dark ages, before the first stars emerged, through to the galaxy-studded cosmos we see today.

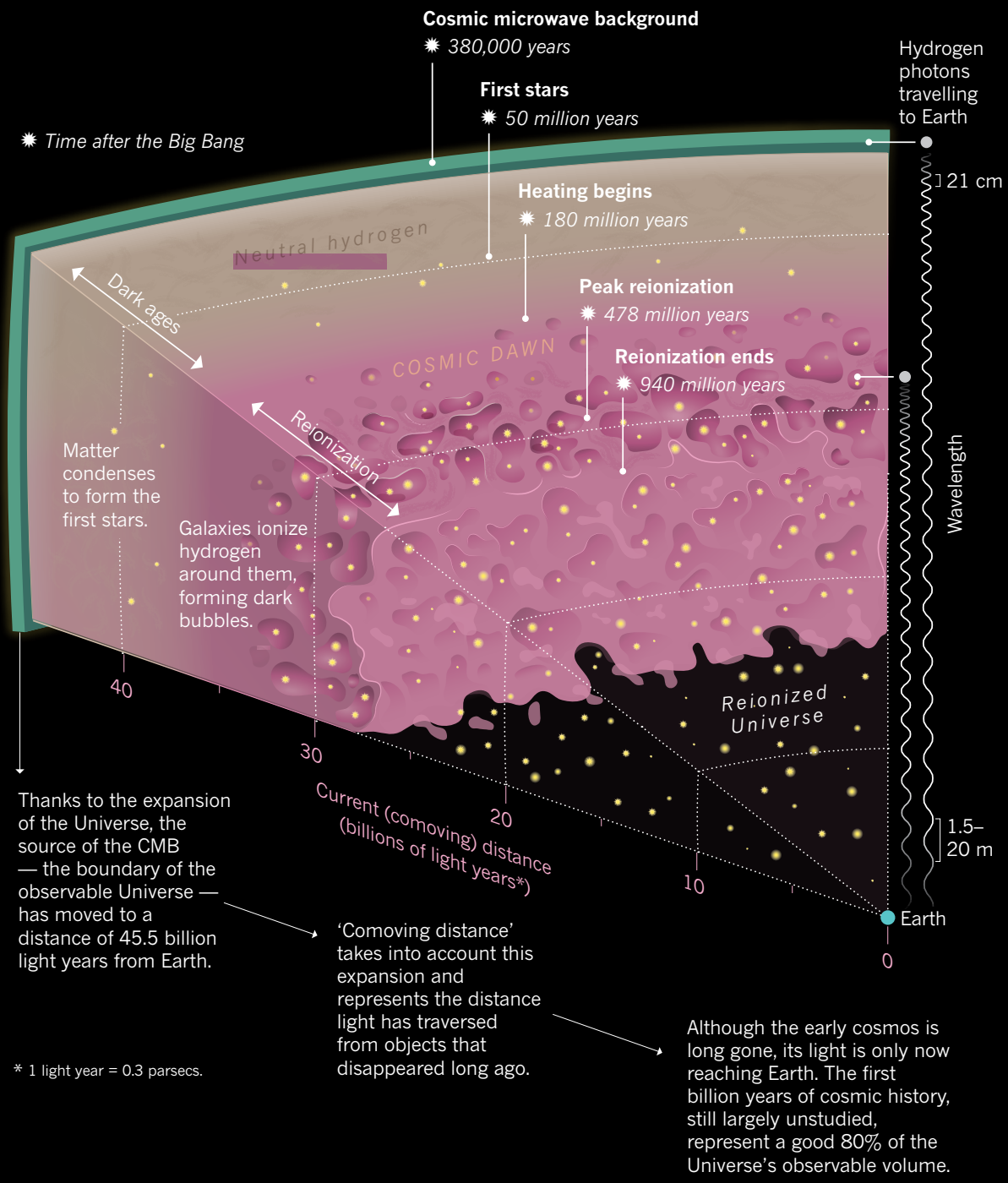

IMPRINT OF AN ATOM

This curve represents the overall brightness of hydrogen's $21-\mathrm{cm}$ signal during the first billion years of the Universe's history.

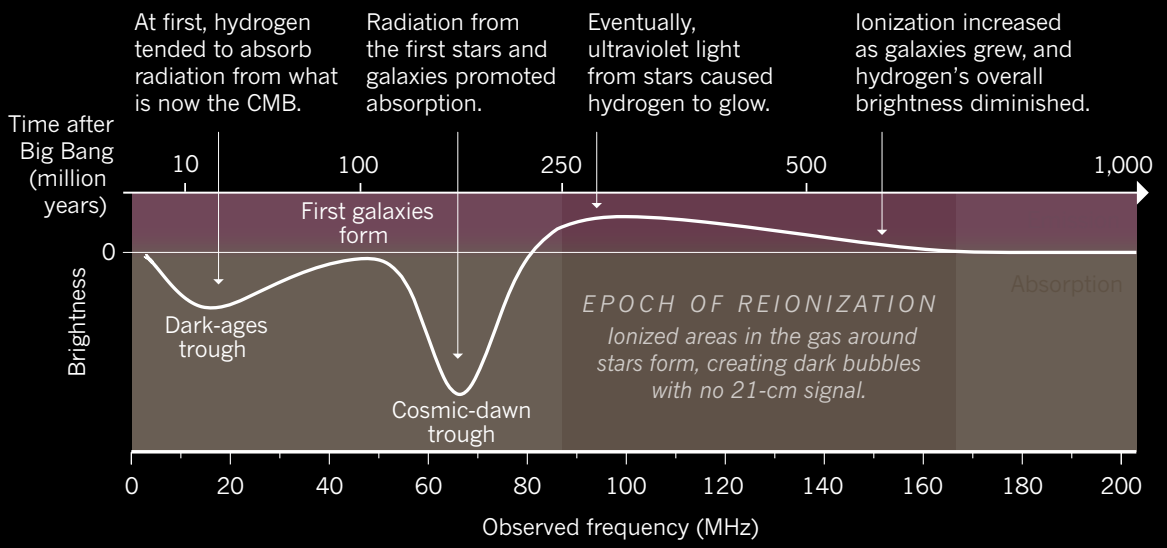

dark matter, nobody has identified exactly what it is. Signals from the EOR would help to indicate whether dark matter consists of relatively sluggish, or 'cold', particles - the model that is currently favoured - or 'warm' ones that are lighter and faster, says Anna Bonaldi, an astrophysicist at the Square Kilometre Array (SKA) Organisation near Manchester, UK. "The exact nature of dark matter is one of the things at stake," she says.

Although astronomers are desperate to learn more about the EOR, they are only now starting to close in on the ability to detect it. Leading the way are radio telescope arrays, which compare signals from multiple antennas to detect variations in the intensity of waves arriving from different directions in the sky.

One of the most advanced tools in the chase is the Low-Frequency Array (LOFAR), which is scattered across multiple European countries and centred near the Dutch town of Exloo. Currently the largest low-frequency radio observatory in the world, it has so far only been able to put limits on the size distribution of the bubbles, thereby excluding some extreme scenarios, such as those in which the intergalactic medium was particularly cold, says Leon Koopmans, an astronomer at the University of Groningen in the Netherlands who leads the EOR studies for LOFAR. Following a recent upgrade, a LOFAR competitor, the Murchison Widefield Array (MWA) in the desert of Western Australia, has further refined those limits in results due to be published soon.

In the short term, researchers say the best chance to measure the actual statistical properties of the EOR - as opposed to placing limits on them - probably rests with another effort called the Hydrogen Epoch of Reionization Array (HERA). The telescope, which consists of a set of 300 parabolic antennas, is being completed in the Northern Cape region of South Africa and is set to start taking data this month. Whereas the MWA and LOFAR are general purpose long-wavelength observatories, HERA's design was optimized for detecting primordial hydrogen. Its tight packing of 14-metre-wide dishes covers wavelengths from $50-250 \mathrm{MHz}$. In theory, that should make it sensitive to the cosmic-dawn trough, when galaxies first began to light up the cosmos, as well as to the EOR (see 'An Earth's-eye view of the early Universe').

As with every experiment of this kind, HERA will have to contend with interference from the Milky Way. The radio-frequency emissions from our Galaxy and others are thousands of times louder than the hydrogen line from the primordial Universe, cautions HERA's principal investigator, Aaron Parsons, a radioastronomer at the University of California, Berkeley. Fortunately, the Galaxy's emissions have a smooth, predictable spectrum, which can be subtracted to reveal cosmological features. To do so, however, radioastronomers must know exactly how their instrument responds to different wavelengths, also known as its systematics. Small changes in the surrounding environment, such 
as an increase in soil moisture or pruning of a nearby bush, can make a difference - just as the quality of an FM radio signal can change depending on where you sit in a room.

If things go well, the HERA team might have its first EOR results in a couple of years, Parsons says. Nichole Barry, an astrophysicist at the University of Melbourne, Australia, and a member of the MWA collaboration, is enthusiastic about its chances: "HERA is going to have enough sensitivity that, if they can get the systematics under control, then boom! They can make a measurement in a short amount of time."

Similar to all existing arrays, HERA will aim to measure the statistics of the bubbles, rather than produce a 3D map. Astronomers' best hope for 3D maps of the EOR lie in the US\$785-million SKA, which is expected to come online in the next decade. The most ambitious radio observatory ever, the SKA will be split between two continents, with the half in Australia being designed to pick up frequencies of $50-350 \mathrm{MHz}$, the band relevant to earlyUniverse hydrogen. (The other half, in South Africa, will be sensitive to higher frequencies.)

\section{CRO-MAGNON COSMOLOGY}

Although arrays are getting bigger and more expensive, another class of $21-\mathrm{cm}$ projects has stayed humble. Many, such as EDGES, collect data with a single antenna and aim to measure some property of radio waves averaged over the entire available sky.

The antennas these projects use are "fairly Cro-Magnon", says CfA radioastronomer Lincoln Greenhill, referring to the primitive nature of the equipment. But researchers spend years painstakingly tweaking instruments to affect their systematics, or using computer models to work out exactly what the systematics are. This is a "masochistic obsession", says Greenhill, who leads the Large-Aperture Experiment to Detect the Dark Ages (LEDA) project in the United States. He often takes solo field trips to LEDA's antennas in Owens Valley, California, to do various tasks. These might include laying a new metal screen on the desert ground beneath the antennas, to act as a mirror for radio waves.

Such subtleties have meant that the community has been slow to accept the EDGES findings. The cosmic-dawn signal that EDGES saw was also unexpectedly large, suggesting that the hydrogen gas that was around 200 million years after the Big Bang was substantially colder than theory predicted, perhaps 4 kelvin instead of 7 kelvin. Since the release of the results in early 2018 , theorists have written dozens of papers proposing mechanisms that could have cooled the gas, but many radioastronomers - including the EDGES team - warn that the experimental findings need to be replicated before the community can accept them.

LEDA is now attempting to do so, as are several other experiments in even more remote and inaccessible places. Ravi Subrahmanyan at the Raman Research Institute in Bengaluru, India, is working on a small, spherical antenna called

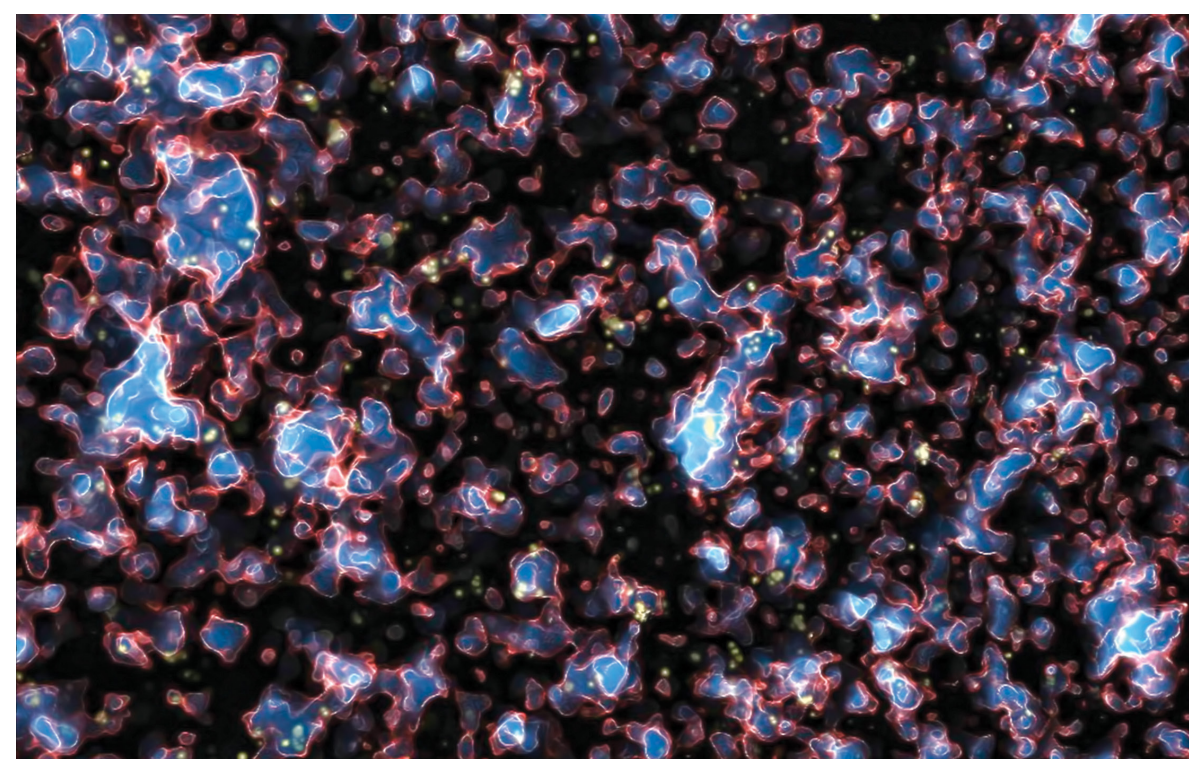

A simulation of the epoch of reionization in the early Universe. Ionized material around new galaxies (bright blue) would no longer emit 21-centimetre radiation. Neutral hydrogen, still glowing at $21 \mathrm{~cm}$, appears dark.

SARAS 2. He and his team took it to a site on the Tibetan Plateau, and they are now experimenting with placing it on a raft in the middle of a lake. With fresh water, "you are assured you have a homogeneous medium below", Subrahmanyan says, which could make the antenna's response much simpler to understand, compared to that on soil.

Physicist Cynthia Chiang and her colleagues at the University of KwaZulu-Natal in Durban, South Africa, went even farther - halfway to Antarctica, to the remote Marion Island - to set up their cosmic-dawn experiment, called Probing Radio Intensity at High-Z from Marion. Chiang, who is now at McGill University in Montreal, Canada, is also travelling to a new site, Axel Heiberg Island in the Canadian Arctic. It has limited radio interference, and the team hopes to be able to detect frequencies as low as $30 \mathrm{MHz}$, which could allow them to detect the dark-ages trough.

At such low frequencies, the upper atmosphere becomes a serious impediment to observations. The best place on Earth to do them might be Dome C, a high-elevation site in Antarctica, Greenhill says. There, the auroras - a major source of interference - would be below the horizon. But others have their eyes set on space, or on the far side of the Moon. "It's the only radio-quiet location in the inner Solar System," says astrophysicist Jack Burns at the University of Colorado Boulder. He is leading proposals for a simple telescope to be placed in lunar orbit, as well as an array to be deployed by a robotic rover on the Moon's surface.

Other, more conventional techniques have made forays into the first billion years of the Universe's history, detecting a few galaxies and quasars - black-hole-driven beacons that are among the Universe's most luminous phenomena. Future instruments, in particular the James Webb Space Telescope that NASA is due to launch in 2021, will bring more of these findings. But for the foreseeable future, conventional telescopes will spot only some of the very brightest objects, and therefore will be unable to do any kind of exhaustive survey of the sky.

The ultimate dream for many cosmologists is a detailed 3D map of the hydrogen not only during the EOR, but all the way back to the dark ages. That covers a vast amount of space: thanks to cosmic expansion, the first billion years of the Universe's history account for $80 \%$ of the current volume of the observable Universe. So far, the best 3D surveys of galaxies which tend to cover closer, and thus brighter, objects - have made detailed maps of less than $1 \%$ of that volume, says Max Tegmark, a cosmologist at the Massachusetts Institute of Technology in Cambridge. Loeb, Tegmark and others have calculated that the variations in hydrogen density before the EOR contain much more information than the CMB does, which so far has been the gold standard for measuring the main features of the Universe. These include its age, the amount of dark matter it contains and its geometry.

Mapping this early hydrogen will be a huge technical challenge. Jordi Miralda-Escudé, a cosmologist at the University of Barcelona in Spain, says that with current technology, it is so challenging as to be a "pipe dream".

But the pay-off of producing such maps would be immense, says Loeb. "The $21-\mathrm{cm}$ signal offers today the biggest data set on the Universe that will ever be accessible to us." -

Davide Castelvecchi is a senior reporter for Nature based in London.

1. Bowman, J. D., Rogers, A. E. E., Monsalve, R. A Mozdzen, T. J. \& Mahesh, N. Nature 555, 67-70 (2018).

2. Zeldovich, Y. B., Kurt, V. G. \& Syunyaev, R. A. [in Russian] Z. Eksp. Teor. Fiz. 55, 278-286 (1968).

3. Loeb, A. J. Cosmol. Astropart. Phys. 2012, 028 (2012)

4. Mao, Y. Tegmark, M., McQuinn, M. Zaldarriaga, M. \& Zahn, O. Phys. Rev. D 78, 023529 (2008). 